\title{
Impact of Long-term Endurance Performance on Muscles Stiffness in Marathon Runners Over 50 Years Old
}

Krzysztof Maćkała ( $\sim$ krzysztof.mackala@awf.wroc.pl )

Department of Track and Field, Wroclaw University of Health and Sport Sciences, Wroclaw

Dariusz Mroczek

Department of Human Motor Skills, Wroclaw University of Health and Sport Sciences, Wroclaw

Paweł Chmura

Department of Sport Team Games, Wroclaw University of Health and Sport Sciences, Wroclaw

Marek Konefał

Department of Human Motor Skills, Wroclaw University of Health and Sport Sciences, Wroclaw

Damian Pawlik

Department of Human Motor Skills, Wroclaw University of Health and Sport Sciences, Wroclaw Jan Chmura

Department of Human Motor Skills, Wroclaw University of Health and Sport Sciences, Wroclaw

\section{Bartłomiej Paleczny}

Department of Physiology and Pathophysiology, Wroclaw Medical University, Wroclaw

\section{Rafał Seredyński}

Department of Physiology and Pathophysiology, Wroclaw Medical University, Wroclaw

Małgorzata Wyciszkiewicz

Department of Physiology and Pathophysiology, Wroclaw Medical University, Wroclaw

Adrianna Nowicka-Czudak

Department of Physiology and Pathophysiology, Wroclaw Medical University, Wroclaw

\section{Wojciech Łopusiewicz}

Department of Physiology and Pathophysiology, Wroclaw Medical University, Wroclaw

\section{Krystyna Chromik}

Department of Human Motor Skills, Wroclaw University of Health and Sport Sciences, Wroclaw

\section{Dorota Adamiec}

Department of Physiology and Pathophysiology, Wroclaw Medical University, Wroclaw

\section{Szczepan Wiecha}

Department of Physical Education and Health in Biala Podlaska, Jozef Pilsudski University of Physical Education in Warsaw Faculty in Biala Podlaska, Biala Podlaska

\section{Piotr Ponikowski}

Department of Heart Diseases, Wroclaw Medical University, Wroclaw 


\section{Beata Ponikowska}

Center for Heart Diseases, University Hospital in Wroclaw, Wroclaw

\section{Research Article}

Keywords: marathon, muscle stiffness, running economy, endurance performance, older-age runners, DOMS

Posted Date: September 2nd, 2021

DOl: https://doi.org/10.21203/rs.3.rs-844791/v1

License: (c) (i) This work is licensed under a Creative Commons Attribution 4.0 International License. Read Full License 


\section{Abstract}

The aim of this research is to evaluate marathon performance and asses the influence of this longdistance running endurance exercise on the changes of muscle stiffness in recreational runners aged 50 + years. Thirty-one male long-distance runners aged 50-73 years participated in the experiment. The muscle stiffness of quadriceps and calves was measured in two independent sessions: the day before the marathon and $30 \mathrm{~min}$ after the completed marathon run using a Myoton device. The $42.195-\mathrm{km}$ run was completed in $4.30,05 \mathrm{~h} \pm 35.12 \mathrm{~min}$, which indicates an intensity of $79.3 \% \pm 7.1 \%$ of HRmax. The long-term, low-intensity running exercise (marathon) in older recreational runners, along with the low level of HRmax and VO2max showed no statistically significant changes in muscle stiffness (quadriceps and calves). There was reduced muscle stiffness, but only in the triceps of the calf in the dominant (left) leg. Moreover, in order to optimally evaluate the marathon and adequately prepare for the performance training programme, we need to consider the direct and indirect analyses of the running economy, running technique, and HRmax and VO2max and DOMS variables. These variables significantly affect the marathon exercise.

\section{Introduction}

Preparing long-distance runners, especially recreational runners and those over 50 , to participate in marathons ${ }^{1}$ requires a rational strategy of training ${ }^{2}$. This mainly applies to developing the runner' motor abilities, technical skills, and probably the two most important actions: tactical skills and adequate dietary supplementation during the marathon itself ${ }^{3-5}$. Modern long-distance training has to allow runners to sustain specific loads of long duration. Therefore, continuous running, and increased fatigue may cause a runner to experience physiological changes that either enhance or diminish their performance or even make it impossible to continue the run ${ }^{6-8}$. Adequate planning of training for marathons involves selecting appropriate training methods, maintaining a rational relationship between training loads, competition loads ${ }^{9}$, and effective recovery, and applying appropriate pre-and postworkout supplementation ${ }^{10}$.

The majority of long-distance training programmes for a marathon are based on regular long, easy runs of between 20 and $40 \mathrm{~km}^{11,12}$. The main purpose of such training is to develop and/or maintain maximum aerobic power, which is the main requirement in order to successfully complete a marathon, regardless of the level of performance or age of the competitors ${ }^{5,13}$. In addition, according to Saunders et $\mathrm{al}^{14}$. long-distance runs are intended to enhance running economy (RE). This teaches the athlete to run at a pace ${ }^{15}$ as efficiently as possible and translates into actual running pace during the competition ${ }^{16,17}$. Several researchers ${ }^{18-20}$ claim that running economy $(\mathrm{RE})$ is an aerobic demand for the maintenance of running and is referred to as the steady-state oxygen uptake (VO2) related to that speed ${ }^{11}$. It is known from practice that after such a long run the athletes experience a considerable amount of muscle damage and soreness ${ }^{18}$, which may adversely affect their muscle overload in the next training session 21. 
Long-distance running competitions are associated with high mechanical stress due to damage to various muscle fibers, metabolic disorders, and muscle fatigue ${ }^{22}$. These elements have been attributed to stress that leads to muscle swelling and delayed muscle soreness (DOMS). It has been shown that disruption of muscle fibers during DOMS, exacerbated by exercise, changes the mechanical properties of the muscles which causes muscle stiffness ${ }^{23}$. Increasing muscle stiffness impairs muscle function and, as a consequence, reduces the body's ability to continue exercising. From a physiological point of view, the level of muscle stiffness is strongly dependent on the size and architecture of the muscles ${ }^{24-26}$ and their specific structural functionality ${ }^{27}$. The physiological cross-sectional area (PCSA) was identified as one of the most important features determining muscle stiffness. Other determinants of stiffness are the type of muscle fibres and the percentage of fast-twitch and slow-twitch fibres, as the number and composition of fibres, which determine the onset of fatigue and, indirectly, post-training stiffness ${ }^{28}$. Multiple training variables can affect muscle stiffness, including the type of muscle work performed, the functionality of the muscle used (flexors vs extensors), and the amount of effort taken until recovery.

There are no data on the level of muscle stiffness after prolonged exercise, especially running. In turn, it is known that in short-term, dynamic exercise, greater muscle damage causes large eccentric contractions, and that large eccentric muscle contractions during PT cause greater muscle damage than concentric ones ${ }^{29,30}$. This causes more delayed-onset soreness in muscles ${ }^{31,32}$.

Whilst the relationship between physiological or anthropometric variables and marathon finish-time have been widely investigated, no study to date has evaluated muscle stiffness's relative contribution to marathon performance. Therefore, despite the dozen marathon investigations, there is a lack of clarity as to the precise determinants of muscle stiffness on marathon performance. Therefore the recipe for success - completion of the marathon - remains somewhat elusive. Assessing muscle stiffness, when combined with other performance indicators previously analysed would benefit runners and coaches looking to improve their marathon performance. Therefore, the aim of this study was to evaluate marathon performance and asses the influence of this long-term running endurance exercise on the changes of muscle stiffness in middle-aged marathon runners. We hypothesise that muscle stiffness will increase with the time that the marathon lasts, no matter what level of training the runner has at the moment.

\section{Material And Methods 2.1. Study design}

The main objective of this study was to evaluate marathon performance and assess its influence on the changes of muscle stiffness in middle-aged marathon runners. The muscle stiffness of quadriceps was measured in two independent sessions: the day before the marathon and $30 \mathrm{~min}$ after the completed marathon. Myoton measurements of each muscle group (12 points) were taken separately for the left and right legs.

\subsection{Participants}


Thirty-one male long distance runners aged $50-73$ years participated in the experiment. Runners estimated their training experience as $10.61 \pm 8.81$ years on average. The average result of the marathon run for the study group was $4.30,05 \mathrm{~h} \pm 35.12 \mathrm{~min}$. All participants were free from any acute illness or chronic disease and did not take regular medication. The main division criterion was that the runners were over 50 years of age, and had participated in at least two marathons in the previous three years. An additional criterion was that all participants were actively training for long-distance running for at least one year. Before signing informed consent forms, the participants were informed about the aim of the experiment and the risk of injury. The study protocol was approved by the local Institutional Ethics Committee (Senacka Komisja ds. Badań Naukowych przy Akademii Wychowania Fizycznego we Wrocławiu). The research was conducted in accordance with the Declaration of Helsinki.

\subsection{Marathon performance}

The 37th PKO Wrocław Marathon (Wrocław, Poland, 19 September 2019) was organised by the City of Wrocław, Poland. Since the beginning of the run, The PKO Wrocław Marathon has been organised by the city of Wroclaw and is considered to be one of the largest running events in Poland. The PKO Wroclaw Marathon takes place annually in the beginning of September. The data on the running time was obtained from the electronic database of the marathon's organisers. It included the number of runners who started and finished the run, the individual identification number of the run, and the place and time of the run for each participant of the marathon. The individual runtime registered in the event was automatically measured using a radio frequency identification chip system. Intermediate times every 5 $\mathrm{km}$ were measured for the experimental group to accurately analyse the variability of their running pace. In addition, the heart rate (HR) was recorded using a monitor (Polar RS300X GPS; Finland) to examine each participant during the marathon run.

\subsection{Study protocol}

\subsubsection{Applied equipment}

The Myoton PRO (Myoton AS, Estonia and Myoton Ltd., London) is a wireless hand-held device that is placed perpendicular to the skin over the muscle being measured. This device was applied under constant preload $(0.18 \mathrm{~N})$ to pre-compress subcutaneous tissues and exert a brief $(15 \mathrm{~ms})$ mechanical tap at a predetermined force $(0.4 \mathrm{~N})$, followed by a quick release, thereby causing dampened oscillations that are recorded by the testing probe (http://www.myoton.com/en/Technology/Technical-specification). The non-neural tone or tension was calculated from the signal spectrum Fmax (fast Fourier transform [FFT] ) and had the frequency $(\mathrm{Hz})$ of the dampened oscillations. Stiffness $(\mathrm{N} / \mathrm{m})$ was characterised by

the muscle's ability to resist an external force that modified its shape ${ }^{33}$. Elasticity was measured by the logarithmic decrement $(\mathrm{log})$ of the dampened oscillations (dissipation of mechanical energy during one oscillation cycle), thus reflecting the ability of the tissue to recover its shape after being deformed ${ }^{34}$.

\subsubsection{Muscle stiffness measurements}




\section{Field experiments}

On the day before the marathon, the first sample of muscle stiffness was collected. The quadriceps muscle of the thigh and the triceps muscle of the calf were measured. All measurements were performed weekly in a designated room. The participants were supine, on their backs, or on their stomachs on a special bed and they rested for 10 min before muscle stiffness measurements were taken. Testing sites on each muscle were located using a tape measure and marked using a skin-safe pen (Fig. 1). A pillow was placed under the head and a special roller pillow was placed under the lower leg to aid relaxation. One series of three single Myoton measurements of each muscle group (12 points) were measured separately for the left and right leg.

\section{Resting recordings}

Immediately after the marathon run, the second sample was collected. All measurements were performed in a designated tent situated past the marathon finish line. Testing sites on each muscle belly were identified using a tape measure and marked using a skin-safe pen. A pillow was placed under the head and a special roller pillow was placed under the lower leg to aid relaxation. Again, a series of three single Myoton measurements of each muscle group (12 points) was taken separately for the left and right legs.

Reliability between trials (within session) for one of the selected muscles (two series of 10 single measurements) of each group was tested using the intraclass correlation coefficient (ICC) model. Domholdt classification scales for interpreting ICCs were used: very high $=1.00-0.90$; high $=0.89-0.70$; moderate $=0.69-0.50$; and low $=0.49-0.26$. ICCs were found for the rectus femoris (ICC, 0.82), biceps femoris (ICC, 0.86), tibialis anterior (ICC, 0.91), and gastrocnemius (ICC, 0.85). The high reliability of the coefficients indicated that the tests resulted in consistent measurements of muscle stiffness among the Marathon runners. This high reliability of measurements confirm earlier experiment carried out on a group of volleyball players by Mroczek et al. ${ }^{35}$

All variables were examined for normal distribution (Shapiro-Wilk test) and homogeneity of variance (Levene's test). Arithmetic means and standard deviations were calculated, and then compared using oneway repeated measures of the analysis of variance (ANOVA). Moreover, Cohen's $d$ was calculated, and the effect sizes it described were in line with the following premises: $\leq 0.35=$ small effect size; $>0.35$ and < $0.65=$ medium effect size, and $\geq 0.65=$ large effect size ${ }^{36}$.

\section{Statistical analysis}

Data were tested for normality using the Shapiro-Wilk test and for homogeneity of variance (Levene's test). Descriptive statistics included the mean, SD, and SE. To compare mean values of the examined variables, repeated measures of one-way ANOVA were used. The independent variable is the time needed to complete the marathon run, whereas the dependent variables were muscle stiffness (MFT, MBS, and 
HR). After a significant main or interaction effect was established, the data were evaluated with a posthoc Fisher's LSD test. The level of statistical significance was set at $p=0.05$. Additionally, Cohen's $d$ was calculated, and the effect sizes were determined: 0.35 for small effect size; 0.35 and 0.65 for medium effect size, and 0.65 for large effect size (13). The relationship between the variables was determined using Pearson's product-moment correlation. Statistical power was set to be $>0.90$ at the level of $p=0.05$. All statistical analyses were made using the STATISTICA ver. 13.1 (StatSoft. Inc., USA) software package.

\section{Results}

The 42.195-km run was completed in $4.30,05 \mathrm{~h} \pm 35.12 \mathrm{~min}$., which indicates an intensity of $79.3 \% \pm 7.1 \%$ of HRmax. The average body height of the marathon runners was $175.61 \pm 5.74 \mathrm{~cm}$, their body weight was $76.17 \pm 7.73 \mathrm{~kg}$, and their BMI was $24.44 \pm 2.32$. A low level of HRmax and VO2max was visible. Similar relationships can be seen in the case of VO2 at the aerobic threshold (VT1) and anaerobic threshold (VT2). The participants percentage of the Wrocław Marathon on VT1 achieved $76.23 \%$ VO2max. In turn, the possibilities at the VT2 threshold were $91.3 \%$ and $84.65 \%$ HRmax (Table 1). 
Table 1

Baseline characteristics of the participants, presented as mean \pm SD

\begin{tabular}{|ll|}
\hline Variables & Mean \pm SD \\
\hline Age & $57.32 \pm 6.25$ \\
\hline Body weight $(\mathrm{kg})$ & $75.36 \pm 7.89$ \\
\hline Height $(\mathrm{cm})$ & $175.61 \pm 5.74$ \\
\hline Body mass index $(\mathrm{kg} / \mathrm{m} 2)$ & $24.44 \pm 2.32$ \\
\hline Training experience & $10.61 \pm 8.81$ \\
\hline HR max & $169.70 \pm 15.71$ \\
\hline VO ${ }_{2}$ max $(\mathrm{ml} / \mathrm{min} / \mathrm{kg})$ & $44.51 \pm 3.63$ \\
\hline VO ${ }_{2}$ VT1 (ml/min/kg) & $33.93 \pm 4.28$ \\
\hline fR-breaths/min (VT1) & $35.81 \pm 6.16$ \\
\hline VE (VT1) L/min & $76.95 \pm 15.32$ \\
\hline HR (VT1) & $142.10 \pm 17.89$ \\
\hline VO2 VT2 (ml/min/kg) & $40.64 \pm 4.28$ \\
\hline fR-breaths/min (VT2) & $45.20 \pm 5.75$ \\
\hline VE (VT2) L/min & $110.12 \pm 17.55$ \\
\hline HR (VT2) & $161.50 \pm 16.19$ \\
\hline
\end{tabular}

In turn, Fig. 2 shows the course of the variability of speed with the division into individual sections (every $5 \mathrm{~km}$ ) and the average HRmax on these sections. It can be seen that the beginning of the drop in speed starts at $12 \mathrm{~km}$ and continues to the end of the run. Along with the decrease in speed, there was a gradual increase in HRmax which also lasted until the end of the marathon.

The analysis of muscle stiffness levels in relation to their subsequent measurements (before and after the marathon) revealed that a significant effect was observed only for the left calf $(F=6.534(1) ; p=0.016$; medium effect size). No significant effect was found for the left quadriceps ( $F=2.393(1) ; p=0.132)$, the right calf $(\mathrm{F}=3.493(1) ; p=0.071)$, or the right quadriceps $(\mathrm{F}=0.876(1) ; p=0.357)$ (Table 2). 
Table 2

Differences in muscle stiffness before and after the marathon (mean \pm standard deviation)

\begin{tabular}{|lllllll|}
\hline Leg & Muscle group & Before marathon & After marathon & F & SSD & Cohen's $\boldsymbol{d}$ \\
\hline \multirow{2}{*}{ Left } & Calf & $302.88 \pm 33.14$ & $292.10 \pm 22.88$ & 6.534 & 0.016 & 0.38 \\
\cline { 2 - 7 } & Quadriceps & $310.43 \pm 29.46$ & $301.92 \pm 38.00$ & 2.393 & 0.132 & 0.25 \\
\multirow{2}{*}{ Right } & Calf & $313.19 \pm 41.86$ & $303.39 \pm 28.29$ & 3.493 & 0.071 & 0.28 \\
\cline { 2 - 7 } & Quadriceps & $294.94 \pm 20.54$ & $297.94 \pm 25.59$ & 0.876 & 0.357 & 0.13 \\
\hline
\end{tabular}

The analysis of the Spearman's rank correlation did not reveal any significant correlation between running speed or HR level, measured over the entire distance divided into $5 \mathrm{~km}$ sections, and muscle stiffness of the quadriceps and triceps calf muscles. On the other hand, significant relationships occurred only between the V02max measurement (measured before the race) and the speed at each of the 5-km sections of the marathon distance and the finish $(p=0.000034$ and $p=0.000239$, respectively).

\section{Discussion}

The aim of this research was to evaluate marathon performance and asses the influence of this longterm running endurance exercise on the changes of muscle stiffness in middle-aged marathon runners. The hypothesis regarding muscle stiffness was not supported, as the current investigation revealed significantly lower levels of stiffness post-marathon for the calf muscles in the left leg $(p=0.016)$. No significant changes were noted with regard to muscle stiffness at the post-marathon assessment in the other two tested muscle groups (quadriceps, in both the left and right lower limbs, and calf in the right limb). Generally, this was very surprising.

The explanation of this phenomenon is likely to be difficult because none of the previously described studies have documented the impact of a prolonged running effort commonly defined as a marathon on muscle stiffness. Additionally, this requires the consideration of indirect analyses of other variables which effect the marathon effort. This approach is also considered difficult because many of the factors to be analysed were not included in this experiment. The reason for this is that many of these variables are difficult to measure without interfering with the running autonomy. However, it has been well described how long-distance running directly impacts running economy $(R E)$ and muscle damage ${ }^{11}$. It seems reasonable to combine all these factors, due to the non-exclusive relationships, to optimally assess the marathon effort and its direct impact on changes in the runner's body after such a long effort. Knowing this may help runners not only to improve their marathon performance, but also to develop an appropriate training programme, which can optimally prepare them to run $42.195 \mathrm{~m}$, regardless of their level: championship, intermediate, or recreational. An important element of such an analysis is the division into sex, but particularly into age categories, with a special emphasis on $50+37$.

Essentially, marathon performance depends on the running economy (RE) in all the word weariness. RE is an 'aerobic demand' to maintain a proper pace: speed over distance. It is defined as the stationary oxygen 
uptake (VO2) associated with this speed ${ }^{18,19}$. Comparing our participants with younger marathon runners aged $43.9 \pm 8.3$, the values of HRmax were lower by an average of $9.2(\mathrm{bpm})$ and 4.29 $(\mathrm{ml} / \mathrm{min} / \mathrm{kg})^{38}$. Larger differences occur in comparison with the group of recreational runners $(63 \pm 32$ $\mathrm{km} /$ week) aged $34 \pm 8$ years. The differences in Hrmax and VO2max are 14.5 (bpm) and VO2max by $18.69(\mathrm{ml} / \mathrm{min} / \mathrm{kg})$, respectively, in favour of the younger runners ${ }^{39}$. Similar relationships can be seen in the case of VO2 at the aerobic threshold (VT1) and anaerobic threshold (VT2). Younger runners are characterised by higher V02 at these thresholds (3.67 for VT1 and 12.86 for VT2) ${ }^{39}$. Despite the lower values of these indicators, the marathon runners studied in Wrocław consumed more oxygen in relation to their abilities than did younger recreational athletes. The percentage of participants of the Wroctaw Marathon on VT1 achieved $76.23 \%$ VO2max, while the competitors studied by Lanferdini et al. ${ }^{39}$ only $59.49 \%$ VO2max. Comparing the capabilities at the VT2 threshold, the results were 91.3 and $84.65 \%$ HRmax, respectively. Despite this, our marathon runners showed a strong relationship between Vo2 max and speed on each subsequent $5-\mathrm{km}$ section (in the range from 0.000034 to 0.000239 ). This confirmed previous research, which found that there is a strong relationship between VO2 max and the level of effort in a marathon run.

It is also obvious that the RE must be associated with the marathon runner's individual running technique, and that this, in turn, depends on the resistance of the runner's body to fatigue and falling running speed. In our experiment, marathon runners began to experience a drop in running speed after $12 \mathrm{~km}$ of a race. From that moment on, a continuous, slow decrease in speed began, which amounted to approx. $5.6 \%$ at the finish line. This did not confirm the reports of Hettinga et al. ${ }^{40}$ that during the late stages of the marathon (the last 10-15 km) a considerable deceleration usually occurs. This affects even world-class runners and is recognised by runners as 'hitting the wall' ${ }^{41}$. This is probably due to the fact that our runners are classified as slow, recreational runners and they were over 50 years of age, so their marathon effort can be defined as prolonged $(4.30,05 \mathrm{~h} \pm 35.12 \mathrm{~min}$.) but of low intensity.

On the other hand, world-class marathon runners have developed training strategies to manage or prevent fatigue and sharp drops in running speed ${ }^{42}$. The studies by Buckalew et al. ${ }^{43}$ and Chan-Roper et al. ${ }^{44}$ regarding the effects of fatigue on running technique showed that technique changed by decreases in step length rather than step frequency. These changes were directly responsible for the decreased speed. Marathon runners are predominantly rear-foot strikers, which is true for both world-class ${ }^{45}$ and recreational long-distance runners ${ }^{46}$. This can be applied to our marathon runners with an indication of the activity of the left leg, and with particular emphasis on the triceps muscle of the calf. They noticed a few disadvantages in this matter. A significant potential biomechanical limitation of landing with a rearfoot strike pattern is that the foot lands in front of the whole body's centre of mass. This increases the braking force and directly impacts the speed, mainly reducing it by the resulting weaker take-off. This negatively influences the step length by shortening it. The second disadvantage of the running technique when fatigue appears is that landing almost the whole foot on the ground during the early stance and then continuing during the main amortisation phase significantly increases contact time. In turn, the high centre of mass is achieved through knee flexion. The greater the knee flexion, the longer the foot-ground 
contact time and the higher the reduction in speed. Additionally, according to Derrick et al. ${ }^{47}$, the presence of fatigue may decrease the utilisation of the stretch shortening mechanism, especially in the hip and knee joints. This causes the knee flexors and extensors to tire more quickly, which results in reduced leg stiffness. Despite this assumption, the relationship between running speed on each $5-\mathrm{km}$ stretch (increasing fatigue with each $\mathrm{km}$ ) and muscle stiffness was not confirmed with no change in muscle stiffness. However, a much greater correlation was found for the triceps muscle of the calf (mean significance level: $p=0.354612$ ). This can be confirmed by the fact that this muscle has a greater functional impact in the running step technique. In turn, it weakens the ground reaction forces, thus significantly extending the contact time ${ }^{48}$. All these elements mean that there is a significant reduction in speed, and the runners thus achieve poor results. In addition, these undesirable factors should be eliminated in training in order to achieve optimal results in the marathon in relation to motor preparation. At the same time, these parameters, which should not weaken the running technique, had a positive effect on muscle stiffness. This did not change after the marathon effort compared to the measurements before the race.

Here another problem arises that was not discussed earlier. It should be assumed that muscle stiffness is partly related to delayed-onset muscle soreness (DOMS). Delayed-onset muscle soreness appears when humans engage in exercise to which they are unaccustomed, or is prolonged in time. It is likely that after a long-term running effort - training for and participating in a run, e.g. a half marathon or marathon runners show significant muscle damage and soreness. Muscle stiffness is associated with delayed muscle soreness. Muscle stiffness is defined as the change in strength divided by the corresponding change in muscle length. This occurs when the change in muscle length is caused by an external factor, e.g. additional external resistance such as uphill running, or by a change in the external load on the muscle - a sustained effort. In other words, the term 'stiffness' describes the resistance of a muscle to a change in length. Therefore, when the muscle is working (eccentric/concentric contraction, e.g. running step) using its entire length, it is able to generate maximum force, and this should automatically increase its stiffness. On the other hand, when the muscle is working, especially in eccentric contraction, with a significantly shorter length (large flexion of the lower limb in the knee joint), its length does not change significantly, which reduces the generation of force and thus reduces the level of its stiffness. This probably happens in marathon runners.

Following this statement, the question arises of whether, with no changes in muscle stiffness after the marathon, there were changes in DOMS, which are mainly manifested by increased muscle soreness and whether they are two independent or mutually exclusive work-related activities. Changes in the mechanical properties of the muscles observed after prolonged physical activity may be associated with increased joint stiffness. In terms of performance, the increased stiffness was found to be associated with increased speed, increased jump velocity, jump height, and running economy (measured by oxygen consumption) ${ }^{49}$. According to Beck et al. ${ }^{37}$, followed by Kerdok et al. ${ }^{50}$, a critical determinant of running economy is the spring-like storage and return of elastic energy from the leg during a stance. 
Here we have to distinguish between two elements - muscle stiffness and joint stiffness - which is often equated with leg spring stiffness. The latter measures the stiffness of the muscle and tendon, but regarding how well a runner is able to recoil the elastic energy generated during ground contact in each stride. Therefore, increases in joint stiffness, mainly by eccentric contraction movement, shorten ground contact ${ }^{50,51}$ will generate more elastic energy. In turn, this indicates an improvement in running economy over time and an increase of delayed-onset muscle soreness.

According to Beck et al. ${ }^{37}$, it can be concluded that the assessment of older runners may be indirectly based on leg stiffness, through reduced tendon stiffness ${ }^{52,53}$, lower active peak ground vertical reaction forces $(\mathrm{GRF})^{51}$, and greater flexion at the knee joint at landing ${ }^{54,55}$. This suggests that leg spring stiffness decreases with age ${ }^{37}$. Did this occur in our marathon runners? Although DOMS was not the subject of the study and we do not have relevant data, it should be assumed that the DOMS was at the tolerance level of the runner's body immediately after the end of the run. The question therefore is how would the DOMS and muscles stiffness behave if measurements were taken several times, e.g. $12 \mathrm{~h}, 24 \mathrm{~h}$, or $48 \mathrm{~h}$ after the marathon? These unknowns were partially answered by Chleboun et al. ${ }^{56}$, who stated that muscle swelling, as one of the factors of DOMS, does not necessarily account for the sudden increase in postexercise stiffness, whatever the determinant of the subsequent muscle stiffness may be.

This is one of the limitations of this study: the absence of subsequent measurements of muscle stiffness, e.g. $12 \mathrm{~h}$ or $24 \mathrm{~h}$ after completing the marathon. This was not due to the technical feasibility of the measurements, but to the personal reasons of the competitors. Such measurements would have also allowed us to observe the changes in DOMS in relation to the delayed changes. Another limitation is the lack of a running technique evaluation on video recording e.g. $15 \mathrm{~km}$ or $40 \mathrm{~km}$ into the race. This would have allowed us to correctly describe the marathoners' running technique and juxtapose it with VO2 in order to evaluate their running economy.

\section{Conclusion}

The long-term, low-intensity running effort (marathon) in older recreational runners, along with a low level of HRmax and VO2max, showed no statistically significant changes in muscle stiffness (quadriceps and calf muscles). There was, however, reduced muscle stiffness, but only in the triceps calf of the dominant (left) leg. Additionally, when we consider the failure to keep an optimal running economy, expressed as a technical disorder (shortened running step, increased ground contact time, lowering the legs in the knee joint) along with increasing fatigue, we can surmise why muscle stiffness did not change post-exercise. Therefore, the hypothesis that muscle mechanical properties and resting tone may change after prolonged exercise was not confirmed in this experiment. In turn, we can rather confirm that this sustained effort may affect the DOMS level by causing an increase in post-exercise muscle soreness.

Moreover, in order to optimally evaluate the marathon run and prepare an adequate training programme, we need to consider a direct and indirect analysis of all the above-mentioned variables, such as the 
running economy, running technique, HRmax and VO2max and DOMS. These variables significantly affect the marathon effort.

\section{Declarations}

\section{Acknowledgements}

This research was financially supported by the Ministry of Science and Higher Education (Poland) / Wroclaw University of Health and Sport Sciences.

\section{Author contributions (names must be given as initials)}

\section{Contributions}

K.M, J.C., D.M, D.P., P.P, conceived and designed the experiments; K.M, J.C.,D.M, D.P., P.C., M.K.,K.CH., performed the experiments; K.M, J.C.,D.M, D.P., P.C., M.K., analysed the data; B.P., R.S., M.W., A.N-C., W.Ł., D.A., S.W., P.P., B.P. interpreted the results; K.M., D.M., drafted and edited paper; all authors critically revised paper and approved the final version of manuscript.

\section{References}

1. Ahmadyar, B., Rust, C. A., Rosemann, T. \& Knechtle, B. Participation and performance trends in elderly marathoners in four of the world's largest marathons during 2004-2011. Springerplus, 4, 465 https://doi.org/10.1186/s40064-015-1254-6 (2015).

2. Laursen, P. B. \& Jenkins, D. G. The scientific basis for high-intensity interval training. Sports medicine, 32, 53-73 (2002).

3. Keogh, A. et al. Prediction equations for marathon performance: A systematic review. International journal of sports physiology and performance, 14, 1159-1169 (2019).

4. Doherty, C. et al. An evaluation of the training determinants of marathon performance: A metaanalysis with meta-regression. Journal of science and medicine in sport, 23, 182-188 (2020).

5. Chmura, J. et al. The Effects of a Marathon Effort on Psychomotor Performance and Catecholamine Concentration in Runners over 50 Years of Age. Applied Sciences, 10, 2067 (2020).

6. Dotan, R. et al. Relationships of marathon running to physiological, anthropometric and training indices. European Journal of Applied Physiology and Occupational Physiology, 51, 281-293 (1983).

7. Alvero-Cruz, J. R. et al. Predictive performance models in long-distance runners: A narrative review. International Journal of Environmental Research and Public Health, 17, 8289 (2020).

8. Keogh, A. et al. The Determinants of Marathon Performance: An Observational Analysis of Anthropometric, Pre-race and In-race Variables. International Journal of Exercise Science, 13, 1132 
(2020).

9. Salinero, J. J. et al. Predicting race time in male amateur marathon runners. J Sports Med Phys Fitness, 57, 1169-1177 https://doi.org/10.23736/S0022-4707.16.06503-8 (2017).

10. Hansen, E. A., Emanuelsen, A., Gertsen, R. M. \& Sørensen, S. S. R. Improved marathon performance by in-race nutritional strategy intervention. International journal of sport nutrition and exercise metabolism, 24, 645-655 (2014).

11. Quinn, T. J., Manley, M. J., Aziz, J., Padham, J. L. \& MacKenzie, A. M. Aging and factors related to running economy. The Journal of Strength \& Conditioning Research, 25, 2971-2979 (2011).

12. Casado, A., Hanley, B., Santos-Concejero, J. \& Ruis-Pérez, L. World-class long-distance running performances are best predicted by volume of easy runs and deliberate practice of short interval and tempo runs. Journal of strength and conditioning research(2019).

13. Arrese, A. L., Izquierdo, D. M. \& Galindo, J. S. Physiological measures associated with marathon running performance in high-level male and female homogeneous groups. International journal of sports medicine, 27, 289-295 (2006).

14. Saunders, P. U., Pyne, D. B., Telford, R. D. \& Hawley, J. A. Factors affecting running economy in trained distance runners. Sports medicine, 34, 465-485 (2004).

15. Angus, S. D. Did recent world record marathon runners employ optimal pacing strategies? Journal of Sports Sciences, 32, 31-45 (2014).

16. Haney, T. A. Jr \& Mercer, J. A. A description of variability of pacing in marathon distance running. International Journal of Exercise Science, 4, 133 (2011).

17. Kipp, S., Kram, R. \& Hoogkamer, W. Extrapolating metabolic savings in running: implications for performance predictions. Frontiers in physiology, 10, 79 (2019).

18. Kyröläinen, H. et al. Effects of marathon running on running economy and kinematics. European journal of applied physiology, 82, 297-304 (2000).

19. Sproule, J. Running economy deteriorates following $60 \mathrm{~min}$ of exercise at $80 \% \mathrm{~V}$ ` $\mathrm{O} 2 \mathrm{max}$. European journal of applied physiology and occupational physiology, 77, 366-371 (1998).

20. Midgley, A. W., McNaughton, L. R. \& Jones, A. M. Training to enhance the physiological determinants of long-distance running performance. Sports Med, 37, 857-880 (2007).

21. Berg, K., Latin, R. \& Coffey, C. Relationship of somatotype and physical characteristics to distance running performance in middle age runners. The Journal of sports medicine and physical fitness, 38 , 253-257 (1998).

22. Joyner, M. J. \& Coyle, E. F. Endurance exercise performance: the physiology of champions. The Journal of physiology, 586, 35-44 (2008).

23. Clarkson, P. M. \& Hubal, M. J. Exercise-induced muscle damage in humans. Am J Phys Med Rehabil, 81, S52-69 https://doi.org/10.1097/01.PHM.0000029772.45258.43 (2002).

24. Brazier, J. et al. Lower extremity stiffness: effects on performance and injury and implications for training. Strength and Conditioning Journal, 112, 103-112 (2014). 
25. Luu, L. A., Zhang, S., Pelland, C. M. \& Blemker, S. S. in American Society of Biomechanics 39th Annual Meeting Columbus.

26. Behrens, M. et al. Plyometric training improves voluntary activation and strength during isometric, concentric and eccentric contractions. Journal of science and medicine in sport, 19, 170-176 (2016).

27. Zierath, J. R. \& Hawley, J. A. Skeletal muscle fiber type: influence on contractile and metabolic properties. PLoS Biol, 2, e348 https://doi.org/10.1371/journal.pbio.0020348 (2004).

28. Seymore, K. D., Domire, Z. J., DeVita, P., Rider, P. M. \& Kulas, A. S. The effect of Nordic hamstring strength training on muscle architecture, stiffness, and strength. Eur J Appl Physiol, 117, 943-953 https://doi.org/10.1007/s00421-017-3583-3 (2017).

29. Kim, J. \& Lee, J. The relationship of creatine kinase variability with body composition and muscle damage markers following eccentric muscle contractions. Journal of exercise nutrition \& biochemistry, 19, 123 (2015).

30. Wertheimer, V., Antekolović, L. \& Matković, B. R. Muscle Damage Indicators after Land and Aquatic Plyometric Training Programmes. Montenegrin Journal of Sports Science and Medicine 7, Ahead of Print(2018).

31. Hody, S., Rogister, B., Leprince, P., Wang, F. \& Croisier, J. L. Muscle fatigue experienced during maximal eccentric exercise is predictive of the plasma creatine kinase (CK) response. Scand J Med Sci Sports, 23, 501-507 https://doi.org/10.1111/j.1600-0838.2011.01413.x (2013).

32. Kanda, K. et al. Eccentric exercise-induced delayed-onset muscle soreness and changes in markers of muscle damage and inflammation. Exercise immunology review19 (2013).

33. Pisano, F. et al. Quantitative measures of spasticity in post-stroke patients. Clin Neurophysiol, 111, 1015-1022 (2000).

34. Mullix, J., Warner, M. \& Stokes, M. Testing muscle tone and mechanical properties of rectus femoris and biceps femoris using a novel hand held MyotonPRO device: relative ratios and reliability. Working Papers in Health Sciences 1, 1-8(2012).

35. Mroczek, D. et al. Effects of Plyometrics Training on Muscle Stiffness Changes in Male Volleyball Players. J Strength Cond Res, https://doi.org/10.1519/JSC.0000000000003074 (2019).

36. Cohen, J. Statistical power analysis for the behavioral sciences (Academic press, 2013).

37. Beck, O. N. et al. Older runners retain youthful running economy despite biomechanical differences. Medicine and science in sports and exercise, 48, 697 (2016).

38. Nikolaidis, P. T. \& Knechtle, B. Age of peak performance in 50-km ultramarathoners-is it older than in marathoners? Open access journal of sports medicine, 9, 37 (2018).

39. Lanferdini, F. J., Silva, E. S., Machado, E. \& Fischer, G. \& Peyré-Tartaruga, L. A. Physiological predictors of maximal incremental running performance. Frontiers in Physiology, 11, 979 (2020).

40. Hettinga, F. J., Edwards, A. M. \& Hanley, B. The science behind competition and winning in athletics: using world-level competition data to explore pacing and tactics. Frontiers in Sports and Active Living, 1, 11 (2019). 
41. Buman, M. P., Brewer, B. W. \& Cornelius, A. E. A discrete-time hazard model of hitting the wall in recreational marathon runners. Psychology of Sport and Exercise, 10, 662-666 (2009).

42. Hanley, B., Bissas, A. \& Merlino, S. Men's and women's World Championship marathon performances and changes with fatigue are not explained by kinematic differences between footstrike patterns. Frontiers in Sports and Active Living, 2, 102 (2020).

43. Buckalew, D. P., Barlow, D. A., Fischer, J. W. \& Richards, J. G. Biomechanical profile of elite women marathoners. Journal of Applied Biomechanics, 1, 330-347 (1985).

44. Chan-Roper, M., Hunter, I., M, K. \& J, W. M., D, L. E. \& S. Kinematic changes during a marathon for fast and slow runners. J Sports Sci Med, 11, 77-82 (2012).

45. Hanley, B., Bissas, A., Merlino, S. \& Gruber, A. H. Most marathon runners at the 2017 IAAF World Championships were rearfoot strikers, and most did not change footstrike pattern. Journal of biomechanics, 92, 54-60 (2019).

46. Larson, P. et al. Foot strike patterns of recreational and sub-elite runners in a long-distance road race. Journal of sports sciences, 29, 1665-1673 (2011).

47. Derrick, T. R., Dereu, D. \& McLean, S. P. Impacts and kinematic adjustments during an exhaustive run. Medicine \& Science in Sports \& Exercise, 34, 998-1002 (2002).

48. Mercer, J. A., Vance, J., Hreljac, A. \& Hamill, J. Relationship between shock attenuation and stride length during running at different velocities. Eur J Appl Physiol, 87, 403-408 https://doi.org/10.1007/s00421-002-0646-9 (2002).

49. Sadeghi, S., Burckhard, S. \& Schmit, C. Respirometry test manual:WEERC laboratory. (2018).

50. Kerdok, A. E., Biewener, A. A., McMahon, T. A., Weyand, P. G. \& Herr, H. M. Energetics and mechanics of human running on surfaces of different stiffnesses. Journal of applied physiology(2002).

51. Bus, S. A. Ground reaction forces and kinematics in distance running in older-aged men. Medicine \& Science in Sports \& Exercise, 35, 1167-1175 (2003).

52. Karamanidis, K. et al. Inevitable joint angular rotation affects muscle architecture during isometric contraction. J Electromyogr Kinesiol, 15, 608-616 https://doi.org/10.1016/j.jelekin.2005.02.001 (2005).

53. Magnusson, S. P., Narici, M. V., Maganaris, C. N. \& Kjaer, M. Human tendon behaviour and adaptation, in vivo. The Journal of physiology, 586, 71-81 (2008).

54. Fukuchi, R. K. \& Duarte, M. Comparison of three-dimensional lower extremity running kinematics of young adult and elderly runners. Journal of sports sciences, 26, 1447-1454 (2008).

55. Kulmala, J. P. et al. Which muscles compromise human locomotor performance with age? Journal of The Royal Society Interface, 11, 20140858 (2014).

56. Chleboun, G. S., Howell, J. N., Conatser, R. R. \& Giesey, J. J. Relationship between muscle swelling and stiffness after eccentric exercise. Medicine and science in sports and exercise, 30, 529-535 (1998). 
Figures

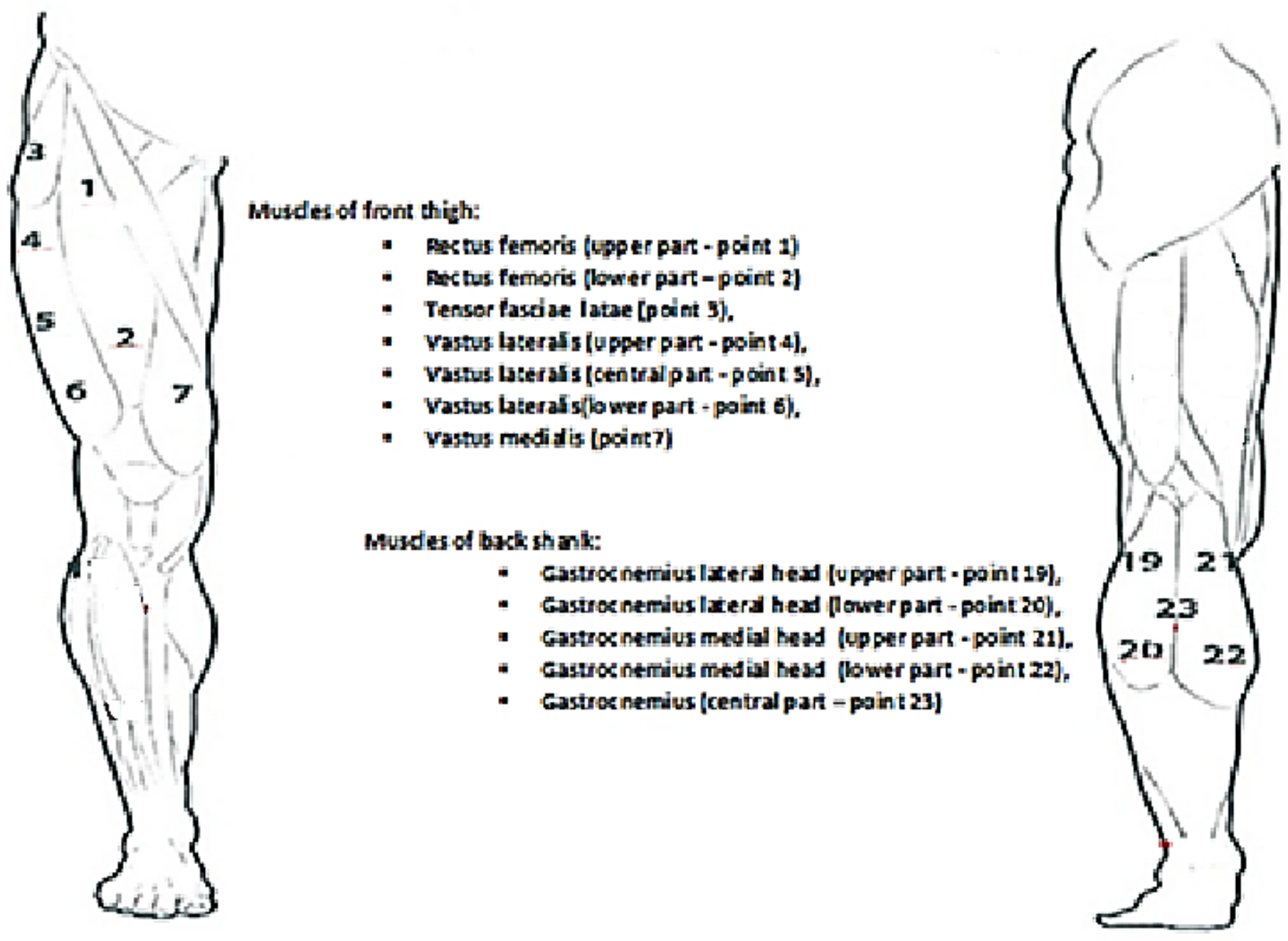

Figure 1

Arrangement of the measurement points of the quadriceps muscle of the thigh and the triceps muscle of the calf 


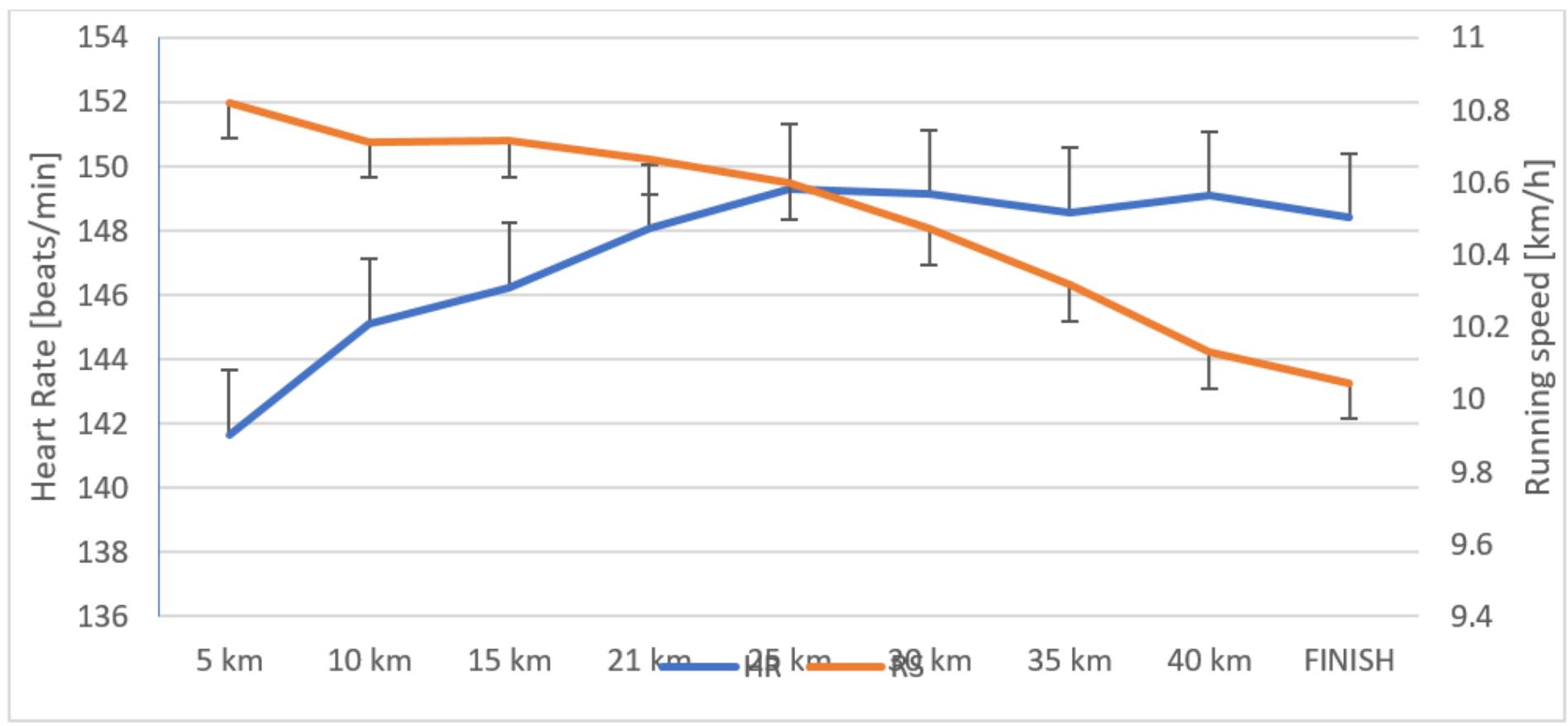

Figure 2

Changes in heart rate [beats $/ \mathrm{min}]$ and running speed $[\mathrm{km} / \mathrm{h}]$ during the marathon (mean $\pm \mathrm{SD}$ ) 